\title{
Fragility of Short-Chain Poly(lactic acid)s Derivatives by Combining Dielectric Spectroscopy and Fast Scanning Calorimetry
}

Sareh Mahmoudian Moghaddam, Blandine Quelennec, Nicolas Delpouve*, Bienvenu Atawa, Elisa Passaglia, Stefano Fiori, Allisson Saiter-Fourcin, Laurent Delbreilh

Dr. S. M. Moghaddam, B. Quelennec, Dr. N. Delpouve, Dr. B. Atawa, Dr. L. Delbreilh, Prof. A. Saiter-Fourcin: Normandie Univ, UNIROUEN Normandie, INSA Rouen, CNRS, Groupe de Physique des Matériaux, 76000 Rouen, France.

Prof. E. Passaglia: Italian National Council for Research - Institute for the Chemistry of OrganoMetallic Compounds (CNR-ICCOM) SS Pisa, via Moruzzi 1, 56124 Pisa, Italy

Dr. S. Fiori: R\&D Department, Condensia Química SA, Barcelona, Spain

*Corresponding author: Nicolas Delpouve

Email: nicolas.delpouve1@univ-rouen.fr

Tel: +33 (0)232955165 / Fax: +33 (0)232955082

Keywords: DSC; cooperativity; glass transition; relaxation; PLA; oligomers

\begin{abstract}
Low-molecular-mass bio-polyesters, such as lactic acid oligomers, constitute a growing category of plasticizers for poly (lactic acid), since they show good compatibility and are respectful of the environment. This study is focusing on the glass transition of a series of oligomers with different molecular weights, with the aim of investigating how their associated dynamics occur. Dielectric and calorimetric results are combined to study the molecular mobility in a large temperature range. In comparison with poly (lactic acid), the oligomers exhibit a lower fragility index and smaller cooperative rearranging regions. Among oligomers, the fragility is clearly dependent on the molecular weight, whereas the variations of cooperativity are subtler.
\end{abstract}




\section{Introduction}

Oligomeric materials represent a growing area of research, ${ }^{[1]}$ in which polymer chemistry is fed by organic synthesis to enable the design of new material properties. Processing biopolymers such as poly (lactic acid) (PLA) with their oligomeric homologues constitutes an interesting way to tune their macroscopic properties, while keeping their environmental friendly character. PLA brittleness is among the main drawbacks limiting its industrial use. Although flexibility improvement was demonstrated from the addition of many plasticizers, the research for efficient additives is still intensive to answer other requirements like decent barrier properties and biocompatibility. ${ }^{[2-3]}$ For the latter, lactic acid oligomers (OLAs), which were characterized by Cicogna et al. ${ }^{[4]}$ in terms of structure, thermal features, and biodegradability once mixed with PLA, showed promises. In the current study, the investigation of OLAs molecular mobility at the glass transition is motivated by its connection with the free volume, ${ }^{[5]}$ an essential structural criterion to understand barrier properties.

The calorimetric signature of the glass transition in PLA and OLAs is easily obtained from modulated temperature differential scanning calorimetry (MT-DSC). To characterize the molecular mobility, one can use the concept of fragility index $m$ introduced by Angell: ${ }^{[6]} m$ depicts how abrupt is the increase of relaxation time $\tau$ when a polymer is cooled down to the glass transition. An abrupt increase of $\tau$, which leads to high values of $m$, is characteristic of fragile polymers, by opposition to strong ones.

$$
m=\left.\frac{d \log _{10}(\tau)}{d\left(T / T_{g}\right)}\right|_{T=T_{g}}
$$

Often, dielectric relaxation spectroscopy (DRS) analyses are performed to plot the temperature dependence of $\tau$, so to obtain $m$. Moreover, it is possible to extend the window in which the molecular mobility can be probed by combining DRS with calorimetric techniques, such as fast 
scanning calorimetry (FSC), and using the concept of fictive temperature $T_{f}$ introduced by Tool. ${ }^{[7]} T_{f}$ depicts how far a polymer in the glassy state is from equilibrium. When the polymer is non-aged, $T_{f}$ is renamed limiting fictive temperature $T_{f}$ ' and directly depends on the cooling rate $\left|\beta_{c}\right|$. This dependence can be adjusted, at least on a limited window, ${ }^{[8-9]}$ by Vogel-FulcherTammann (VFT) equation (2), similarly to the temperature dependence of $\tau$. To improve $m$ estimation accuracy, data issued from FSC can therefore be superimposed to the DRS results, using the same VFT parameters: pre-exponential factor $\tau_{0}$, strength parameter $D$ and Vogel temperature $T_{V}$.

$$
\tau=\tau_{0} \exp \left(\frac{D T_{V}}{T-T_{V}}\right)
$$

The plasticizing efficiency of oligomers increases with the decrease of their chain length, presumably because the free volume increases. Thus, the idea of this study is to combine FSC and DRS to provide a very accurate description of the molecular mobility at the glass transition for amorphous PLA and OLAs, and then to estimate the size of the cooperative rearranging regions related to the free volume, quantity directly linked to the barrier properties. ${ }^{[5,10]}$ The characteristic cooperativity volume $\xi^{3} T \alpha$ at the dynamic glass transition temperature $T_{\alpha}$ has been calculated from MT-DSC data by applying Donth's equation: ${ }^{[11]}$

$$
\xi_{T_{\alpha}}^{3}=\frac{\left(\frac{1}{C p_{\text {glass } T \alpha}}-\frac{1}{C p_{\text {liquid } T \alpha}}\right) k_{B} T_{\alpha}^{2}}{\rho \delta T^{2}}
$$

Where $\delta T$ is the mean temperature fluctuation related to the dynamic glass transition of one CRR, $k_{B}$ is the Boltzmann constant, $\rho$ is the density and $C_{p}$ is the heat capacity at constant pressure. 


\section{Experimental}

Oligomers of D-/L-lactic acid (Glyplast ${ }^{\circledR}$ OLAs, code $\mathrm{OLA} M_{w}$, where $M_{w}$ is the weight average molecular mass in $\mathrm{g} / \mathrm{mol}$, being equal to 1400,1900 , or 2700 ) were synthesized and supplied by Condensia Química SA® ${ }^{[4]} M_{w}$ values were obtained by size exclusion chromatography (Agilent Technologies 1200 Series instrument equipped with a degasser, an isocratic HPLC pump, a refractive index (RI) detector, and two PLgel $5 \mu \mathrm{m}$ MiniMIX-D columns), ${ }^{[4]}$ and did not change after thermal treatment. PLA pellets were provided by Natureworks ${ }^{\circledR}$ (grade 4042D having 4.3\% D-, and 95.7\% L-lactic acid, named PDLLA in the rest of the manuscript), with $M_{w}=188 \mathrm{~kg} / \mathrm{mol}$. Films were obtained by applying 2 bars pressure on melted pellets, initially dried at $50{ }^{\circ} \mathrm{C}$ for several hours, under steel plates of Specac $\AA$ manual hydraulic press. Amorphous samples were obtained by quenching from the melt, in liquid nitrogen for OLAs, in cold water for PDLLA.

MT-DSC experiments were performed at $2 \mathrm{~K} / \mathrm{min}$ under $50 \mathrm{~mL} / \mathrm{min}$ nitrogen flow gas in a TA® DSC Q2000 apparatus equipped with a refrigerating cooling system. The calibration was performed using indium for temperature and energy, and using sapphire for heat capacity. The modulation parameters were 60 s period and $\pm 0.318 \mathrm{~K}$ amplitude. In line with previous works, the deconvolution procedure, ${ }^{[12]}$ and phase lag correction, ${ }^{[13]}$ were done.

DRS analyses were performed using an Alpha Analyzer from Novocontrol® technologies. Spectra were collected from -150 up to $100{ }^{\circ} \mathrm{C}$ in a frequency range of $10^{-1}-2 * 10^{6} \mathrm{~Hz}$. PDLLA film was placed between $30 \mathrm{~mm}$ gold parallel electrodes. OLAs were placed on interdigitated electrodes (20 mm sensor diameter and 0.001 loss factor accuracy). DRS responses were analyzed using the Havriliak-Negami complex function. 
FSC experiments were performed in Mettler Toledo® Flash DSC1 apparatus equipped with a Huber® TC100 intracooler. Each MultiSTAR UFS 1 MEMS® chip sensor was conditioned before the low thickness sample was positioned on it. The static thermal lag was corrected from the response of a gallium standard. The dynamic thermal lag was corrected as described elsewhere. ${ }^{[8]}$ The sample mass was estimated from the amplitude of the glass transition, by assuming that the heat capacity step of the amorphous system was the same than the one determined from MT-DSC. ${ }^{[8]}$ The atmosphere was continuously flushed with nitrogen (20 $\mathrm{mL} / \mathrm{min})$.

\section{Results and discussion}

Figure 1 shows the MT-DSC responses of amorphous PDLLA and OLAs recorded in the glass transition range. From MT-DSC, the observation of the glass transition is facilitated. One can separate the kinetic events which sometimes occur in its temperature domain from its calorimetric signature itself, i.e., the heat capacity increment, which manifests in the reversing heat capacity signal. The results are presented in Table 1. As expected, the glass transition $T_{g}$ shifts to lower temperatures with the decrease of the chain length. The heat capacity step $\triangle C p$ at $T_{g}$ slightly increases from 0.51 (PDLLA) up to 0.60 (OLA1400) J/(g.K). 


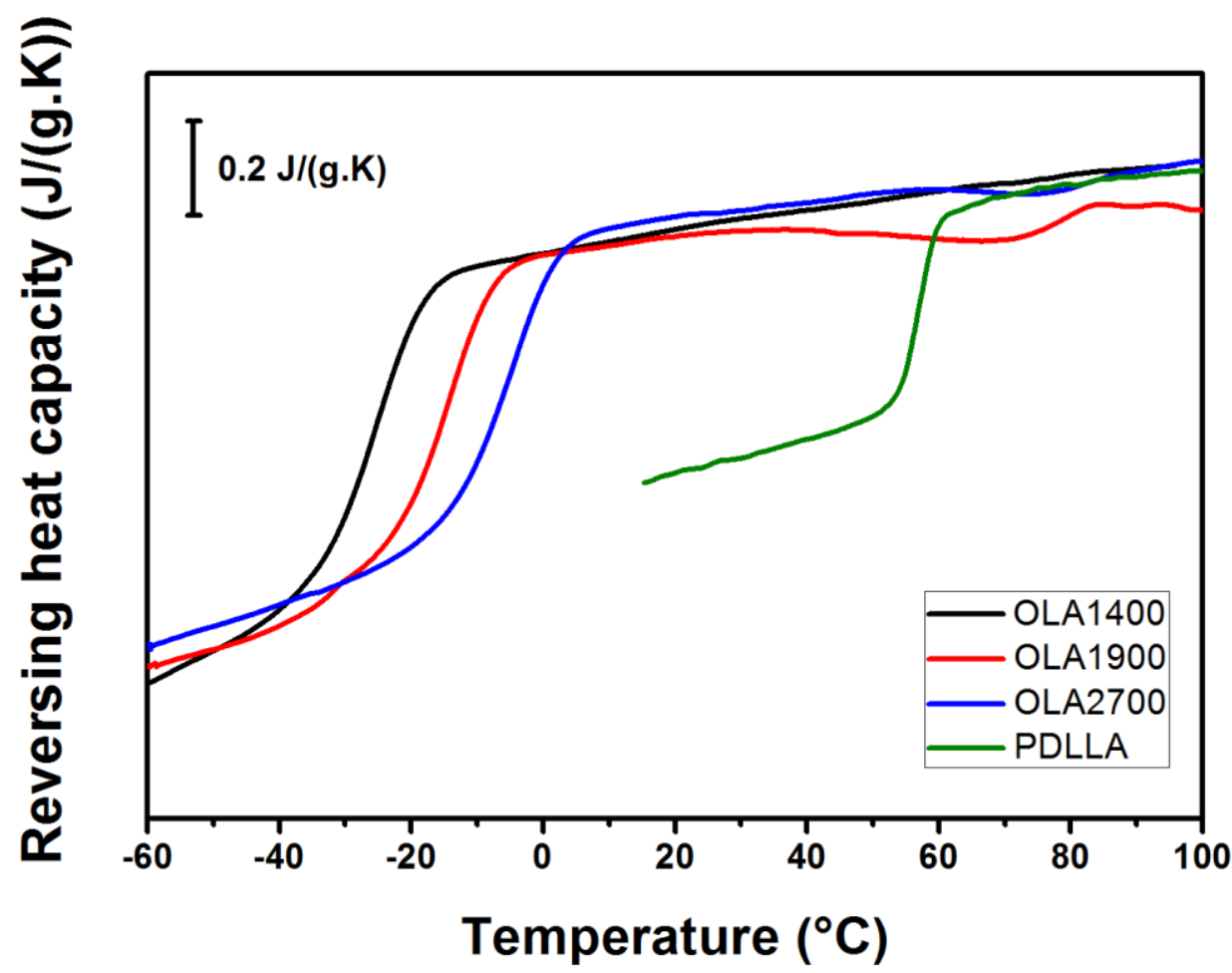

Figure 1: MT-DSC reversing heat capacity signals recorded in the glass transition range for amorphous PDLLA and OLAs.

Table 1: Glass transition characteristic parameters for PDLLA and OLAs from calorimetric and dielectric measurements.

\begin{tabular}{|c|c|c|c|c|c|c|c|c|}
\hline & \multicolumn{2}{|c|}{ MT-DSC } & \multicolumn{6}{|c|}{$\mathbf{F S C}+\mathbf{D R S}$} \\
\hline & $\begin{array}{l}T_{g} \\
(\mathrm{~K})\end{array}$ & $\begin{array}{c}\Delta C p \\
(\mathrm{~J} /(\mathrm{g} . \mathrm{K}))\end{array}$ & $\log C$ & $\log \left[\left(1 / \tau_{0}\right)\left(\mathrm{s}^{-1}\right)\right]$ & $D$ & $\begin{array}{l}T_{V} \\
(\mathrm{~K})\end{array}$ & $\begin{array}{c}T_{g(\tau=100 \mathrm{~s})} \\
(\mathrm{K})\end{array}$ & $m$ \\
\hline PDLLA & 330.0 & 0.51 & 0.87 & 12.13 & 3.29 & 297.0 & 327.0 & 155 \\
\hline OLA2700 & 268.0 & 0.55 & 0.30 & 14.01 & 7.18 & 222.5 & 266.0 & 99 \\
\hline OLA1900 & 259.5 & 0.57 & 0.35 & 14.91 & 9.70 & 206.5 & 257.5 & 85 \\
\hline OLA1400 & 246.5 & 0.60 & 0.47 & 17.90 & 19.14 & 172.5 & 244.5 & 68 \\
\hline
\end{tabular}



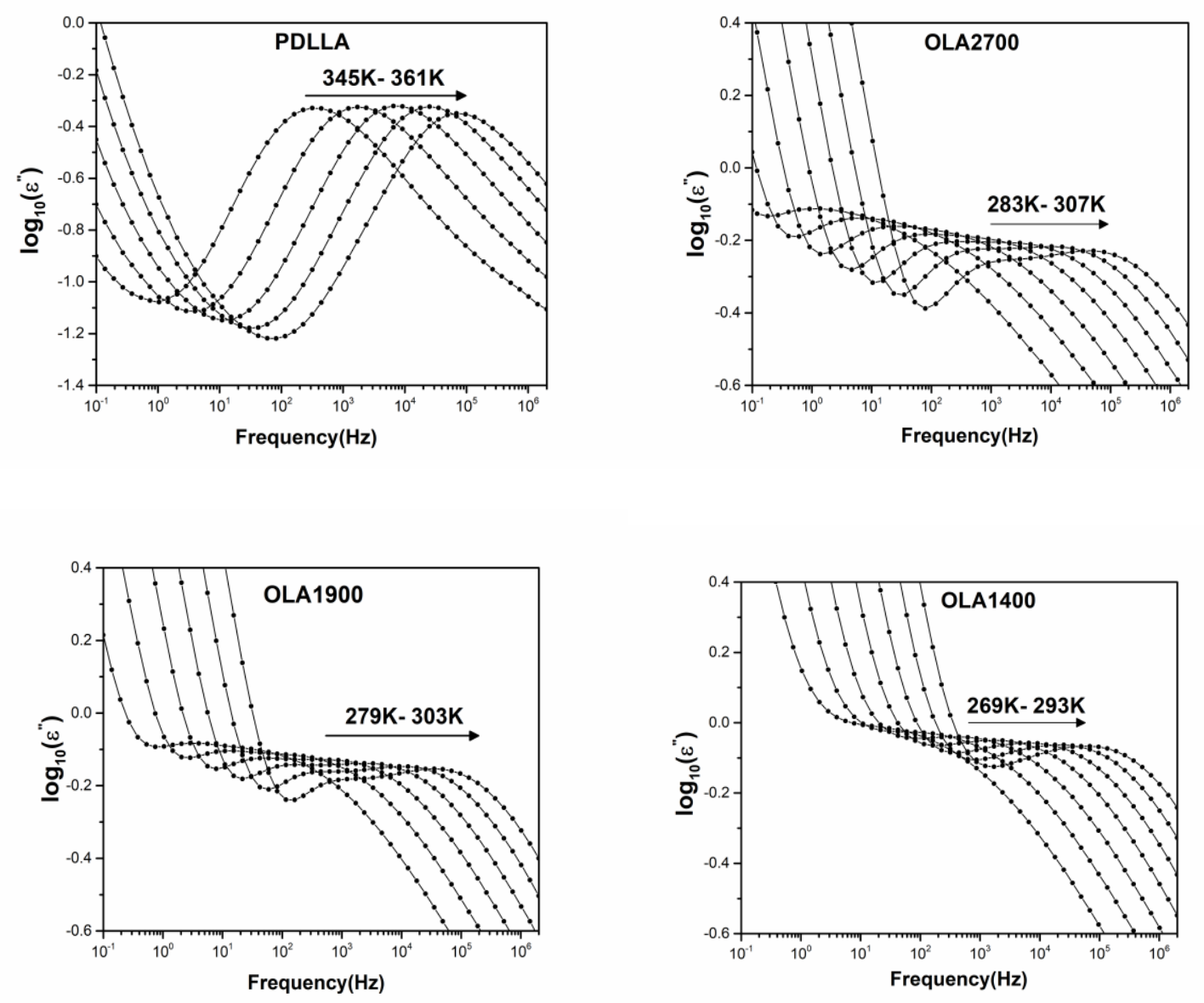

Figure 2: Frequency dependence of the imaginary part of complex permittivity $\varepsilon$ '” at several temperatures in logarithmic scale for PDLLA, OLA2700, OLA1900, and OLA1400.

In Figure 2 the dielectric response of the imaginary part of complex permittivity noted $\varepsilon$ '” is presented for all the samples in the glass transition domain, at various temperatures, as a function of frequency. For each temperature, the slope in the low frequency domain is due to the conductivity, whereas the signature of the glass transition is recorded at high frequency. As expected, this signature shifts to higher frequencies with increasing temperature. Finally, one can observe a shouldering close to the conductivity slope, likely due to a slower relaxation mode. 


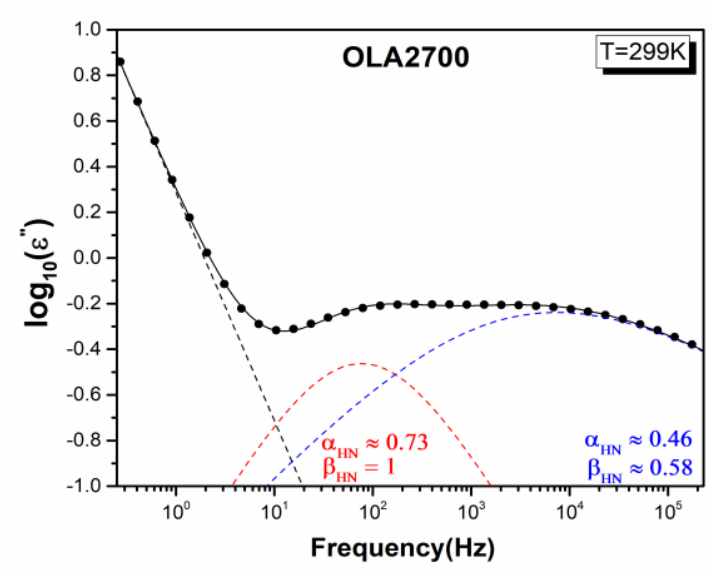

Figure 3: Fitting procedure used to investigate the segmental relaxation region with a conductivity contribution (black dashed line) and two Havriliak-Negami complex functions: one for the low frequency process (red dashed line) and another for the $\alpha$-relaxation (blue dashed line). The black solid line is the final fitting. The Havriliak-Negami shape parameters $\alpha_{H N}$ and $\beta_{H N}$ are also reported. This example concerns the dielectric response of OLA2700 recorded at $299 \mathrm{~K}$.

Figure 3 shows the analytical fitting procedure of OLA2700 dielectric response $\varepsilon$ '” at 299 K. The relaxation time $\tau$ was extracted using a conductivity $\sigma$ term and two Havriliak-Negami contributions. The low frequency contribution (in red) might be the signature of the normalmode, as reported for PLA, ${ }^{[14-15]}$ related to the fluctuation and orientation of the end-to-end chain, ${ }^{[16]}$ but this is not ascertained and goes beyond the scope of the study. The high frequency contribution (in blue), that is the contribution of interest in this study, from which $\tau$ is obtained, is associated with the main relaxation of polymer chains, or $\alpha$-relaxation. The general expression for the Havriliak-Negami complex function is given below.

$$
\varepsilon^{*}(\omega)=-i\left(\frac{\sigma}{\omega \varepsilon_{0}}\right)^{n}+\sum_{k} \frac{\Delta \varepsilon_{k}}{\left[1+\left(i \omega \tau_{k}\right)^{\alpha_{k}}\right]^{\beta_{k}}}+\varepsilon_{\infty}
$$


Where $\omega$ is the angular pulsation, $k$ is the number of contributions for the fitting procedure (equal to 2 in the present study), $n$ is a fitting parameter related to the conductivity slope (black dashed line), $\Delta \varepsilon$ is the dielectric strength, $\alpha_{H N}$ and $\beta_{H N}$ are the symmetric and asymmetric broadenings of the complex dielectric function respectively, $\varepsilon_{\infty}$ is the permittivity at high frequency of the relaxation process, $\varepsilon_{0}$ is the vacuum permittivity. The procedure is extended to the measurements performed at different temperatures in the glass transition range to obtain the temperature dependence of the $\alpha$-relaxation time. The results are presented in Figure 4 showing the temperature dependence for all the relaxation processes detected from DRS measurements. The DRS signatures of local relaxations are given in Supporting Information (Figure SI1).

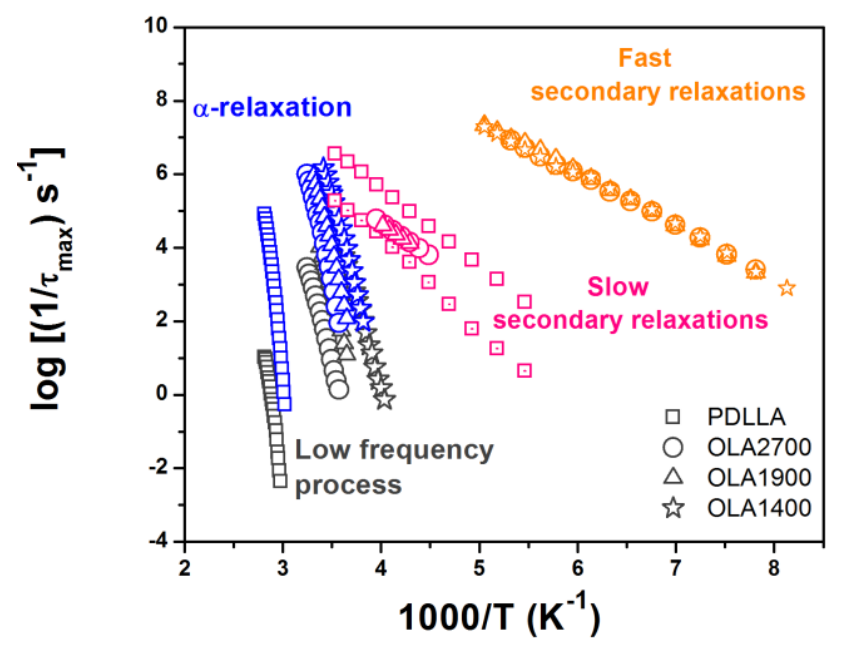

Figure 4: Relaxation map including all the relaxation processes recorded from DRS measurements for PDLLA, OLA2700, OLA1900, and OLA1400.

One can notice first that PDLLA and OLAs exhibit different local relaxation processes. In PDLLA, two relaxations are recorded in the same temperature range. On the other hand, OLAs undergo a common fast local process at temperatures situated farther below. Besides, the 
detection of a slow local process is more difficult. It has only been recorded for OLA2700 and OLA1900, still on a very narrow temperature range. Furthermore, the temperature dependences of both $\alpha$-relaxation and low frequency process are obviously steeper for PDLLA than those of oligomers.

Figure 5 shows FSC curves of PDLLA and OLAs. The analyses were performed at constant heating rate $\beta_{h}=1500 \mathrm{~K} / \mathrm{s}$ for various $\left|\beta_{c}\right|$. The glass transition appears as an endothermic step with a superimposed recovery peak used to calculate the dependence of $T_{f}^{\prime}$ with $\left|\beta_{c}\right|$. By decreasing the cooling rate, the peak increases in amplitude and shifts towards higher temperatures. The area matching method,${ }^{[17]}$ which allows calculating the limiting fictive temperature $T_{f}$ ' is presented in Supporting Information (Figure SI2). Data from FSC and DRS are plotted in Figure 6 and superimposed by using Frenkel-Kobeko-Reiner (FKR) equation (5), using same $D, \tau_{0}$ and $T_{V}$ fit parameters, and applying a simple constant logarithmic shift $\log C$ (Fitting parameters given in Table 1). $T_{g_{(\tau=100 \mathrm{~s})}}$ is the glass transition temperature arbitrary defined as the temperature for which $\tau$ and the experimental time scale are of the same order, i.e., about 100 s.

$$
\log \left(\left|\beta_{c}\right|\right)=\log (1 / \tau)+\log C=\log \left(1 / \tau_{0}\right)-\left(\frac{D T_{V}}{\ln \left(T-T_{V}\right)}\right)+\log C
$$



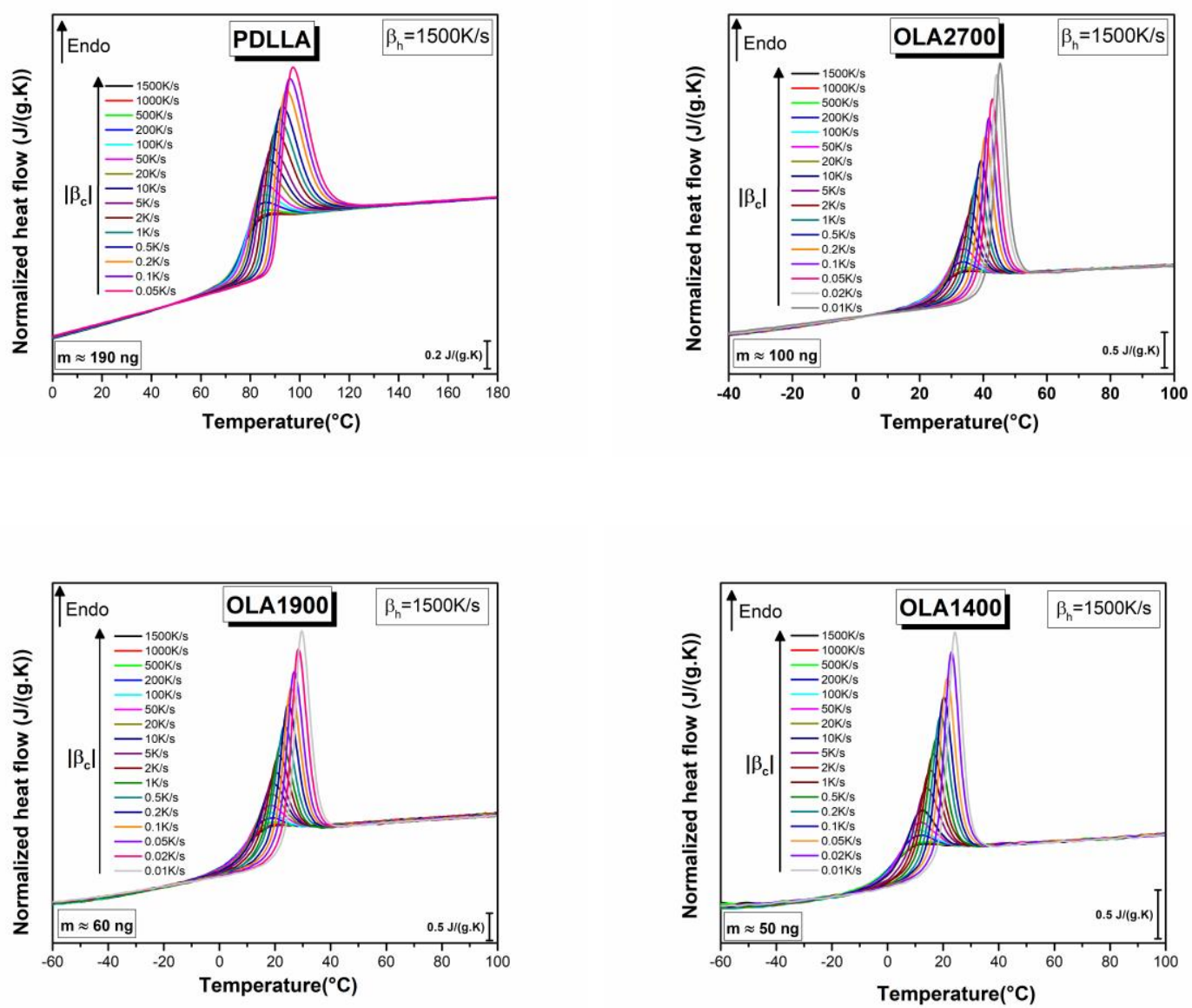

Figure 5: FSC normalized heat flow of amorphous PDLLA and OLAs for various cooling rate $\left|\beta_{c}\right|$ (ranging from $0.01 \mathrm{~K} / \mathrm{s}$ up to $1500 \mathrm{~K} / \mathrm{s}$ ) at constant heating rate $\beta_{h}=1500 \mathrm{~K} / \mathrm{s}$. The sample masses have been estimating by comparing the $\Delta C p$ step values obtained by MT-DSC and FSC. ${ }^{[8]}$

MT-DSC results are also added. It is worth mentioning that the validity of this approach can be questioned since a decoupling between vitrification and molecular mobility has recently been evidenced. ${ }^{[18-19]}$ Nevertheless, as can be seen in Figure 6, dielectric and calorimetric data superimpose well in the present study. In consistence with previous studies, ${ }^{[5]}$ PDLLA exhibits a fragile behavior $(m=155)$. On the other hand, oligomers exhibit lower $m$, which decreases from 99 (OLA2700) down to 68 (OLA1400) with the decrease of $M_{w}$. The dependence of the segmental dynamics on the chain length has been the focus of several studies. ${ }^{[20-24]} \mathrm{A}$ correlation between the fragility index and the chain length is frequently observed. On the other 
hand, some systems show no dependence of the fragility index on the molecular weight. ${ }^{[20]}$ According to Santangelo and Roland, ${ }^{[21]}$ only polymers which exhibit a high cooperativity length at the glass transition, that is the case of PDLLA, ${ }^{[25]}$ show a significant decrease of $m$ with the decrease of $M_{w}$.

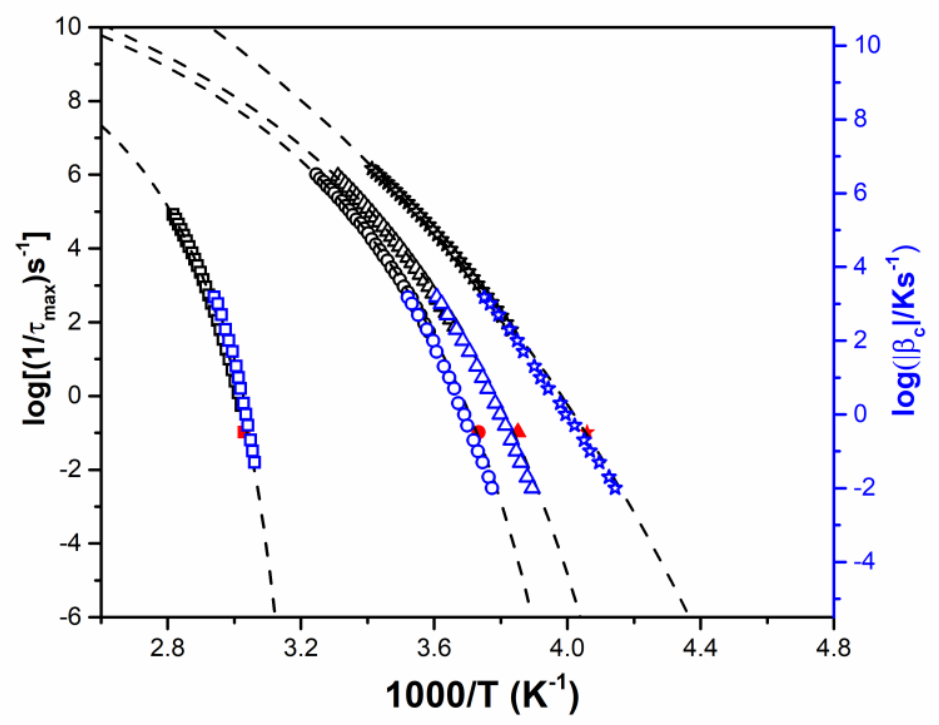

Figure 6: Temperature dependence of the relaxation rate $\left(1 / \tau_{\max }\right)$ (left coordinate) and cooling rate $\left|\beta_{c}\right|$ dependence of $T_{f}$ ' (right coordinate), determined from DRS (black symbols), MT-DSC (red symbols) and FSC (blue symbols) respectively, for PDLLA (squares), OLA2700 (circles), OLA1900 (triangles) and OLA1400 (stars). Dashed line is VFT fit to all data obtained from FSC+DRS. The glass transition temperature $T_{g}$ from MT-DSC is defined at $\tau=10$ s which approximately corresponds to a 60 s period.

In Figure 7, $m$ is plotted as a function of $\xi^{3} T \alpha$. Data obtained from the analysis of other PLAs have been added (results given in Figures SI3, SI4 and SI5 of Supporting Information). There is a global consistency between the decreases of both fragility and cooperativity, which can be interpreted as the result of the increase of the free volume with the reducing of $M_{w}$. By focusing 
on the results obtained for the oligomers, the correlation between $m$ and $\xi_{T \alpha}^{3}$ is less obvious since they exhibit close cooperativity volumes and significantly different fragility indexes. One can wonder why the cooperativity length of oligomers is poorly impacted by the molecular weight. This result might be linked to their chemical structure. They carry an end-chain group that increases their plasticizing efficiency. ${ }^{[4]}$ Determining the influence of this group on the segmental dynamics will require further investigations. .

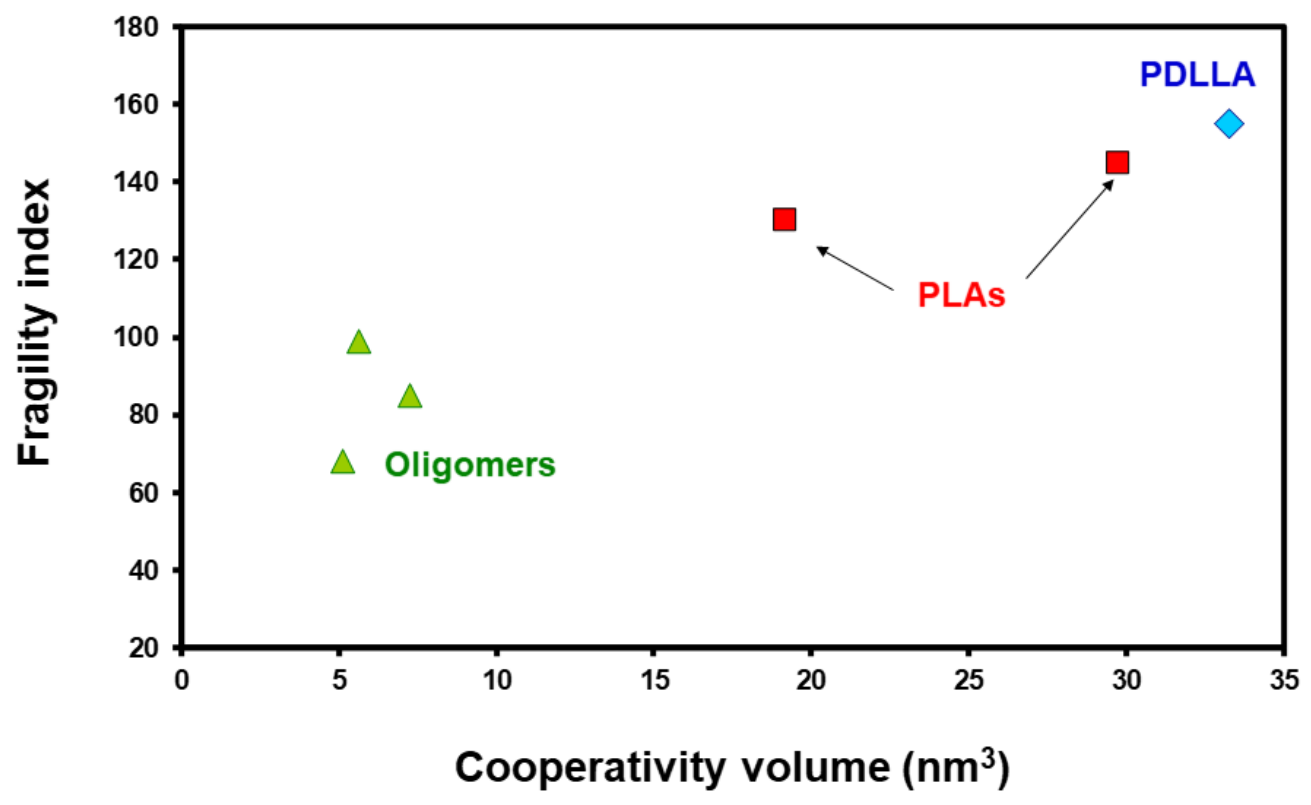

Figure 7: Fragility index as a function of the cooperativity volume for PDLLA and OLAs. Additional data were obtained from investigations on Poly(L-lactide)s (PLAs) with number average molecular weight equal to 5000 and $10000 \mathrm{~g} / \mathrm{mol}$ (Sigma Aldrich ${ }^{\circledR}$; product number 764590 and 765112; CAS Number: unknown and 0026161422 respectively).

\section{Conclusion}

The tracking of the segmental relaxation dynamics by combining calorimetric and dielectric investigations has provided a relaxation map from which the fragility has been estimated without much uncertainty. The global agreement between cooperativity and fragility variations 
is sufficiently convincing to interpret the difference of segmental dynamics between the lactic acid oligomers and poly (lactic acid) by a fall of cooperativity with the reducing of the chain length. This assumption is not fully ascertained when it comes to explaining the variations of fragility among oligomers only, since the cooperativity volume is about the same. Free volume measurements would therefore be of interest to complement the results of this study. 


\section{References}

[1] B. van Genabeek, B. A. G. Lamers, C. J. Hawker, E. W. Meijer, W. R. Gutekunst, B. V. K. J. Schmidt, J. Polym. Sci. 2021, 59, 373.

[2] L. C. Llanes, S. H. Clasen, A. T. N. Pires, I. P. Gross, Eur. Polym. J. 2021, 142, 110112.

[3] J. Tan, T. Lu, R. Li, S. Zhang, W. Liu, X. Zhu, J. Zhang, J. Xin, ACS Sustain. Chem. Eng. 2019, 7, 15957.

[4] F. Cicogna, S. Coiai, C. De Monte, R. Spiniello, S. Fiori, M. Franceschi, F. Braca, P. Cinelli, S.M.K. Fehri, A. Lazzeri, W. Oberhauser, E. Passaglia, Polym. Int. 2017, 66, 761.

[5] S. Araujo, N. Delpouve, S. Domenek, A. Guinault, R. Golovchak, R. Szatanik, A. Ingram, L. Delbreilh, E. Dargent, Macromolecules 2019, 52, 6107.

[6] C. A. Angell, J. Non-Cryst. Solids 1985, 73, 1.

[7] A. Q. Tool, J. Am. Ceram. Soc. 1946, 29, 240.

[8] J. E. K. Schawe, Thermochim. Acta 2015, 603, 128.

[9] A. Dhotel, B. Rijal, L. Delbreilh, E. Dargent, A. Saiter, J. Therm. Anal. Calorim. 2015, 121, 453.

[10] A. Saiter, JM Saiter, R Golovchak, M Shpotyuk, O Shpotyuk, J. Phys. Condens. Matter 2009, 21, 075105 .

[11] E. Hempel, G. Hempel, A. Hensel, C. Schick, E. Donth, J. Phys. Chem. B 2000, 104, 2460. [12] A. A. Lacey, D. M. Price, M. Reading, in Modulated Temperature Differential Scanning Calorimetry, Vol. 6 (Eds: M. Reading, D. J. Hourston), Springer, Dordrecht, Netherlands, 2006, Chap. 1, pp. 1-81.

[13] S. Weyer, A. Hensel, C. Schick, Thermochim. Acta 1997, 304-305, 267.

[14] M. Mierzwa, G. Floudas, J. Dorgan, D. Knauss, J Wegner, J. Non-Cryst. Solids 2002, 307-310, 296.

[15] J. Ren, O. Urakawa, K. Adachi, Macromolecules 2003, 36, 210. 
[16] K. Adachi, T. Kotaka, Prog. Polym. Sci. 1993, 18, 585.

[17] C. T. Moynihan, A. J. Easteal, M. A. DeBolt, J. Tucker, J. Am. Ceram. Soc. 1976, 59, 12.

[18] X. Monnier, D. Cangialosi, Phys. Rev. Lett., 2018, 121, 137801.

[19] X. Monnier, D. Cangialosi, B. Ruta, R. Busch, I. Gallino, Sci. Adv. 2020, 6, eaay1454.

[20] L. Andreozzi, C. Autiero, M. Faetti, M. Giordano, F. Zulli, Philos. Mag. 2008, 88, 4151.

[21] P. G. Santangelo, C. M. Roland, Macromolecules 1998, 31, 4581.

[22] C. Dalle-Ferrier, A. Kisliuk, L. Hong, G. Carini Jr., G. D'Angelo, C. Alba-Simionesco, V. N. Novikov, A. P. Sokolov, J. Chem. Phys. 2014, 145, 154901.

[23] K. Xing, M. Tress, P.-F. Cao, F. Fan, S. Cheng, T. Saito, A. P. Sokolov, Macromolecules 2018, 51,8561

[24] D. L. Baker, M. Reynolds, R. Masurel, P. D. Olmsted, J. Mattsson, (Preprint) arXiv.org, arXiv:1911.13278, version1, submitted: November 2019.

[25] B. Rijal, L. Delbreilh, A. Saiter, Macromolecules 2015, 48, 8219. 
Sareh Mahmoudian Moghaddam, Blandine Quelennec, Nicolas Delpouve*, Bienvenu Atawa, Elisa Passaglia, Stefano Fiori, Allisson Saiter-Fourcin, Laurent Delbreilh

Fragility of Short-Chain Poly(lactic acid)s Derivatives by Combining Dielectric Spectroscopy and Fast Scanning Calorimetry

\section{Table of contents:}

Segmental relaxation dynamics of lactic acid oligomers depend on the molecular weight, as evidenced from the spectacular variations regarding the fragility index that has been calculated by combining the temperature dependence of the relaxation time (dielectric measurements) with the cooling rate dependence of the limiting fictive temperature (calorimetric measurements). The investigated systems are also characterized by a low cooperativity at the glass transition.

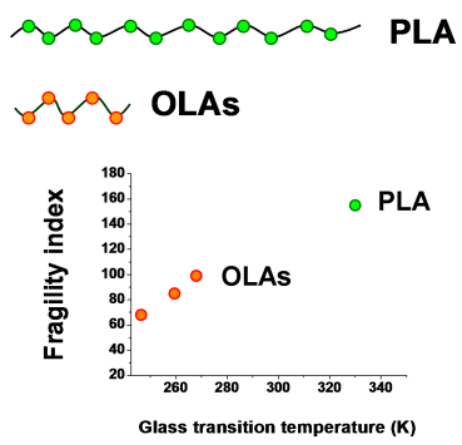




\section{Supporting Information}

\section{Fragility of Short-Chain Poly(lactic acid)s Derivatives by Combining Dielectric Spectroscopy and Fast Scanning Calorimetry}

Sareh Mahmoudian Moghaddam, Blandine Quelennec, Nicolas Delpouve*, Bienvenu Atawa, Elisa Passaglia, Stefano Fiori, Allisson Saiter-Fourcin, Laurent Delbreilh

Dr. S. M. Moghaddam, B. Quelennec, Dr. N. Delpouve, Dr. B. Atawa, Dr. L. Delbreilh, Prof. A. Saiter-Fourcin: Normandie Univ, UNIROUEN Normandie, INSA Rouen, CNRS, Groupe de Physique des Matériaux, 76000 Rouen, France.

Prof. E. Passaglia: Italian National Council for Research - Institute for the Chemistry of OrganoMetallic Compounds (CNR-ICCOM) SS Pisa, via Moruzzi 1, 56124 Pisa, Italy

Dr. S. Fiori: R\&D Department, Condensia Química SA, Barcelona, Spain

*Corresponding author: Nicolas Delpouve

Email: nicolas.delpouve1@univ-rouen.fr

Tel: +33 (0)232955165 / Fax: +33 (0)232955082 
As shown in Figure SI.1, PDLLA exhibits two local relaxation processes in close temperature domains. OLAs exhibit a different behaviour. All share the same fast local relaxation which occurs between 128 and 158K, farther below local relaxations of PDLLA. A slower process can be clearly observed only for OLA1900 and OLA2700. This one is recorded between 233 and $248 \mathrm{~K}$.
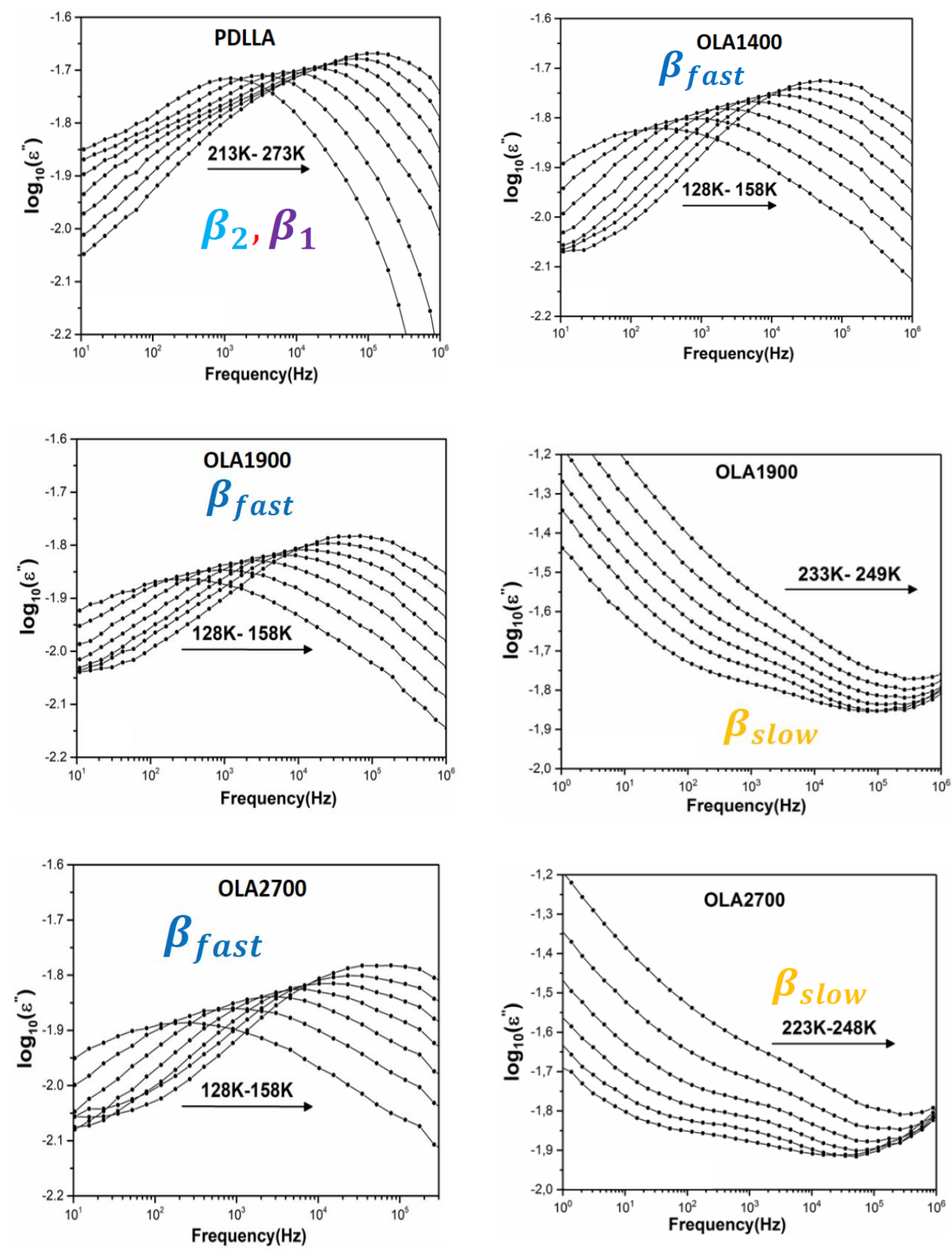

Figure SI.1: DRS signatures of local relaxation processes recorded for PDLLA and OLAs. 
In Figure SI.2 the area matching method [1] is presented, which allows calculating the limiting fictive temperature $T_{f}$ ' from the following equation:

$$
\int_{T_{f^{\prime}}}^{T_{2}}\left(C_{p, l}-C_{p, g}\right) d T=\int_{T_{1}}^{T_{2}}\left(C_{p}-C_{p, g}\right) d T
$$

Where $C p, g$ and $C p, l$ are the heat capacity of the glass and the liquid respectively, $C p$ is the heat capacity of the material, $T_{1}$ and $T_{2}$ are two arbitrary temperatures, chosen in the glass and the liquid like states respectively, in such a way that $C p=C p, g$ at $T_{1}$ and $C p=C p, l$ at $T_{2}$. In this study, the signal used to apply this method was the FSC heat flow, having been normalized to the heating rate $\beta_{h}$ and the sample mass, to be expressed in unit equivalent to the heat capacity one. The procedure is extended to the measurements performed at different cooling rates $\left|\beta_{c}\right|$ to obtain the cooling rate dependence of the limiting fictive temperature.

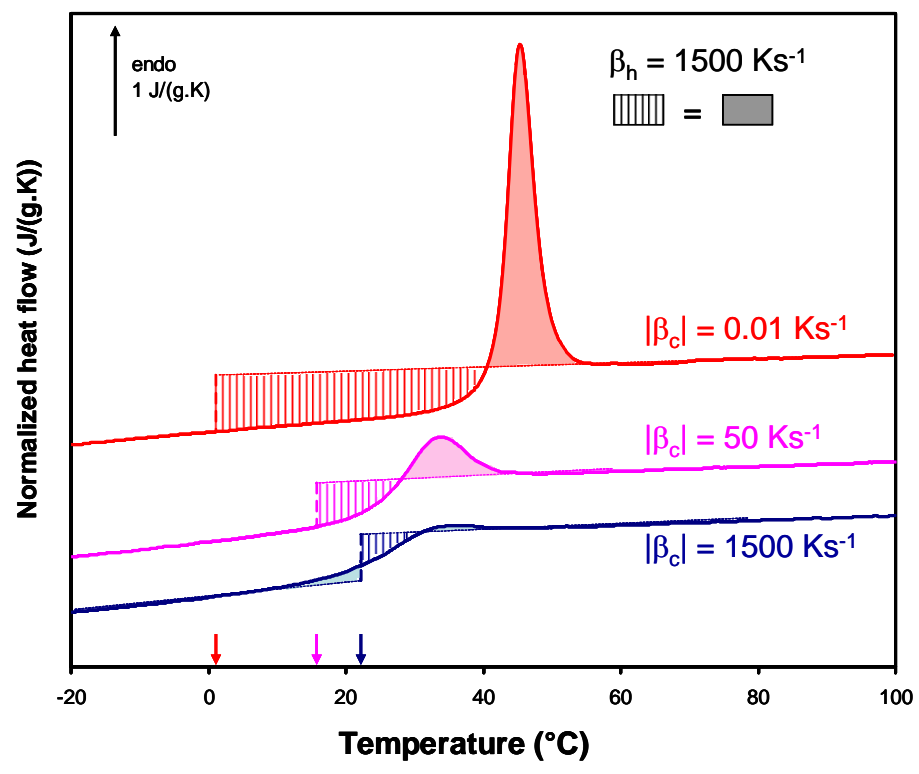

Figure SI.2: Principle of the area matching method used to determine the limiting fictive temperature $T_{f}$ ' for various cooling rates $\left|\beta_{c}\right|$ at constant heating rate $\beta_{h}=1500 \mathrm{~K} / \mathrm{s}$. Dashed lines and arrows mark $T_{f}$. Dotted lines are used to show temperature dependence of $\mathrm{Cp}$ in glass and liquid like states.

[1] C.T. Moynihan, A.J. Easteal, M.A. DeBolt, J. Tucker, Dependence of the fictive temperature of glass on cooling rate, J. Am. Ceram. Soc. 59 (1976) 12-16. https://doi.org/10.1111/j.11512916.1976.tb09376.x. 
In Figure SI.3, MT-DSC results for PLA5000 and PLA10000 are shown. PLA10000 exhibits similar $T_{g}$ and $\Delta C p$ than PDLLA, i.e. $330 \mathrm{~K}$ and $0.5 \mathrm{~J} /(\mathrm{g} . \mathrm{K})$ respectively. The glass transition of PLA5000 is 4K lower, but its heat capacity step is the same than PDLLA and PLA10000.

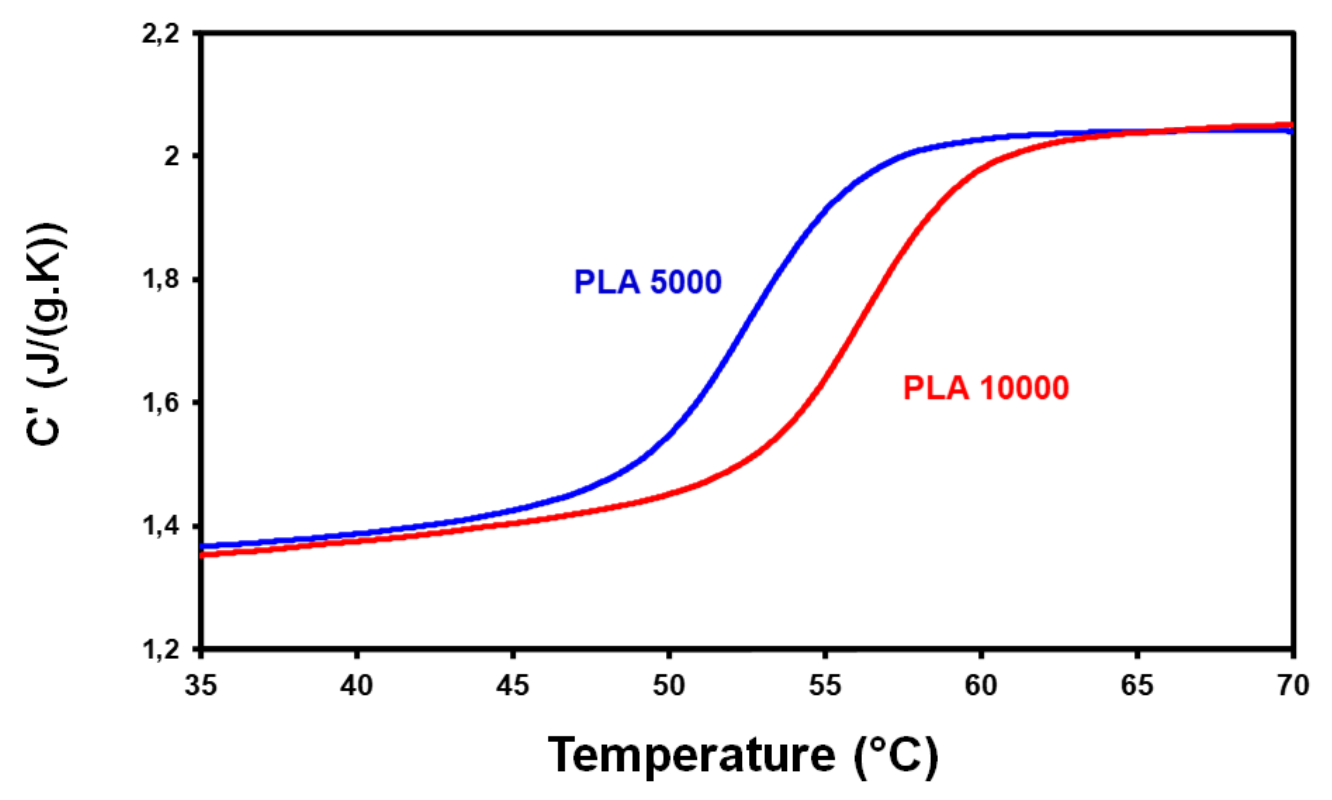

Figure SI3: MT-DSC reversing heat capacity signal recorded in the glass transition range for amorphous PLA5000 and PLA10000. 
In Figure SI.4, the dielectric response $\varepsilon$ ' is presented for PLA5000 and PLA10000 in the glass transition domain.
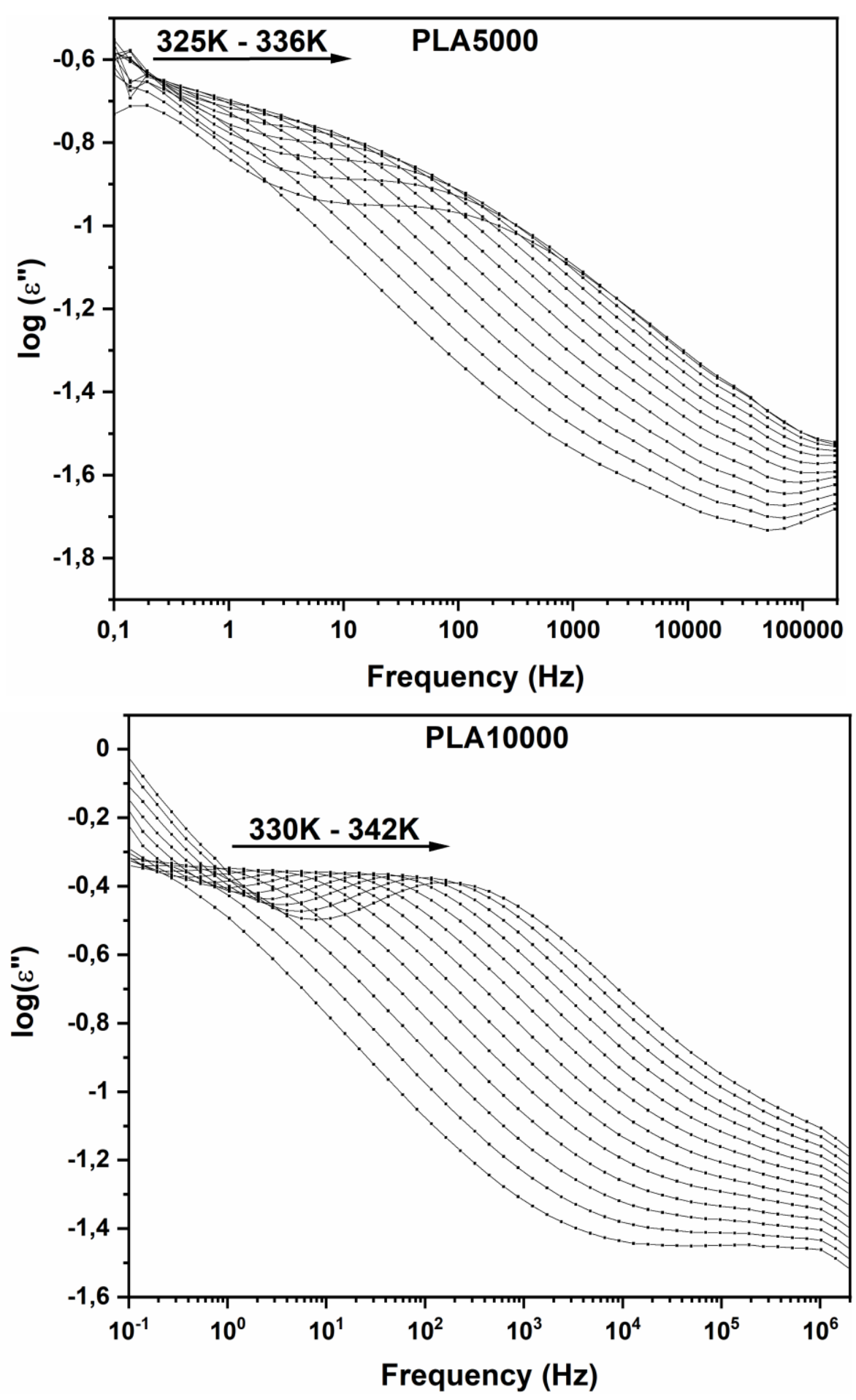

Figure SI4: Frequency dependence of the imaginary part of the complex permittivity $\varepsilon$ "' at several temperatures in logarithmic scale for PLA5000 and PLA10000. 
In Figure SI.5, the relaxation maps obtained consecutively to DRS investigations on PLA5000 and PLA10000 are presented. In comparison with PDLLA, the fragility of both systems is slightly lesser: $m=145$ and $m=130$ for PLA10000 and PLA5000 respectively.

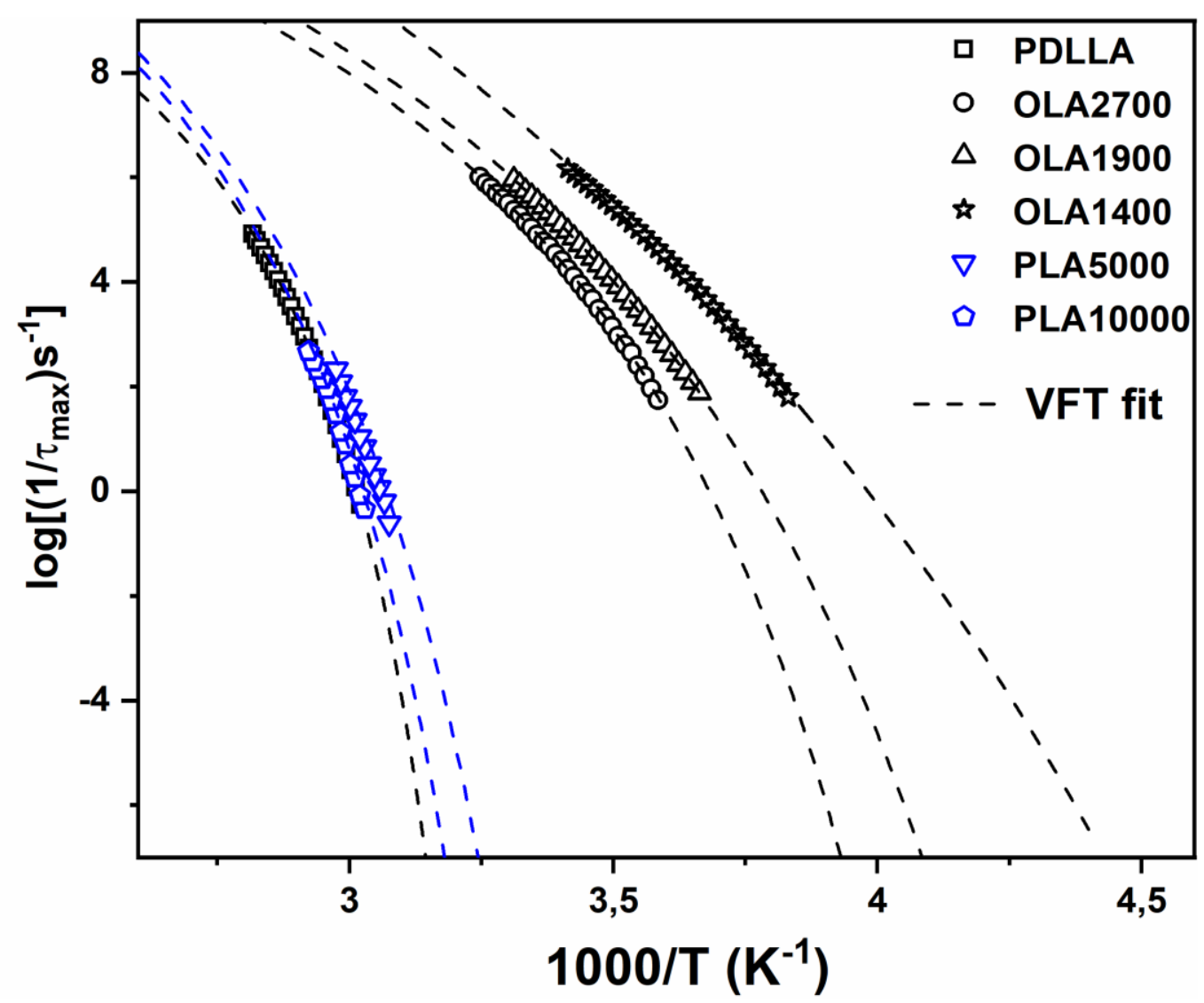

Figure SI.5: Temperature dependence of the relaxation rate $\left(1 / \tau_{\max }\right)$ determined from DRS for PLA5000 (blue triangles) and PLA10000 (blue pentagons). Results obtained for PDLLA (black squares), OLA2700 (black circles), OLA1900 (black triangles) and OLA1400 (black stars) are added. The dashed curve is the VFT fit of the experimental data. 